ARTICLE

\title{
A flexible electron-blocking interfacial shield for dendrite-free solid lithium metal batteries
}

Hanyu Huo ${ }^{1,2}$, Jian Gao ${ }^{3}$, Ning Zhao ${ }^{4}$, Dongxing Zhang (D) $^{5}$, Nathaniel Graham Holmes ${ }^{1}$, Xiaona Li , Yipeng Sun ${ }^{1}$, Jiamin Fu', Ruying $\mathrm{Li}^{1}$, Xiangxin Guo (i) ${ }^{4 凶} \&$ Xueliang Sun (i) ${ }^{1 凶}$

Solid-state batteries (SSBs) are considered to be the next-generation lithium-ion battery technology due to their enhanced energy density and safety. However, the high electronic conductivity of solid-state electrolytes (SSEs) leads to Li dendrite nucleation and proliferation. Uneven electric-field distribution resulting from poor interfacial contact can further promote dendritic deposition and lead to rapid short circuiting of SSBs. Herein, we propose a flexible electron-blocking interfacial shield (EBS) to protect garnet electrolytes from the electronic degradation. The EBS formed by an in-situ substitution reaction can not only increase lithiophilicity but also stabilize the Li volume change, maintaining the integrity of the interface during repeated cycling. Density functional theory calculations show a high electrontunneling energy barrier from Li metal to the EBS, indicating an excellent capacity for electron-blocking. EBS protected cells exhibit an improved critical current density of $1.2 \mathrm{~mA} \mathrm{~cm}^{-2}$ and stable cycling for over $400 \mathrm{~h}$ at $1 \mathrm{~mA} \mathrm{~cm}-2\left(1 \mathrm{mAh} \mathrm{cm}^{-2}\right)$ at room temperature. These results demonstrate an effective strategy for the suppression of Li dendrites and present fresh insight into the rational design of the SSE and Li metal interface.

\footnotetext{
${ }^{1}$ Department of Mechanical and Materials Engineering, University of Western Ontario, London, ON N6A 5B9, Canada. ${ }^{2}$ State Key Laboratory of High Performance Ceramics and Superfine Microstructure, Shanghai Institute of Ceramics, Chinese Academy of Sciences, 200050 Shanghai, China. ${ }^{3}$ State Key Laboratory of Organic-Inorganic Composites, Beijing University of Chemical Technology, 100029 Beijing, China. ${ }^{4}$ College of Physics, Qingdao University, 266071 Qingdao, China. ${ }^{5}$ Department of Chemistry, University of Western Ontario, London, ON N6A 5B9, Canada. ${ }^{凶}$ email: xxguo@qdu.edu.cn; xsun9@uwo.ca
} 


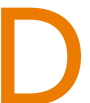
ue to the rapid development of portable devices and electric vehicles, current lithium-ion batteries cannot meet future requirements for energy density, cycle life, and safety ${ }^{1}$. Solid-state batteries (SSBs) have received much attention for their potential as next-generation batteries ${ }^{2}$. Solidstate electrolytes (SSEs) paired with a Li metal anode and a highvoltage cathode not only enhance energy density but also improve safety through the elimination of flammable liquid electrolytes.

Many SSEs have been reported, including lithium phosphorus oxynitride $(\mathrm{LiPON})^{3}$, sulfide-type ${ }^{4}$, sodium superionic conductor (NASICON)-type ${ }^{5,6}$, perovskite-type ${ }^{7}$, halide-type ${ }^{8}$, and garnettype ${ }^{9}$. Among them, garnet-type $\mathrm{Li}_{7} \mathrm{La}_{3} \mathrm{Zr}_{2} \mathrm{O}_{12}$ (LLZO) is promising because of its excellent chemical/electrochemical stability with $\mathrm{Li}$ metal and its high ionic conductivity at room temperature ${ }^{10,11}$. LLZO has a high shear modulus ( $\left.\sim 55 \mathrm{GPa}\right)$ and $\mathrm{Li}$ transference number $(\sim 1)$, and was therefore predicted to prevent Li dendrite growth based on Sand's theory ${ }^{12-15}$. Unfortunately, numerous studies have reported garnet electrolytes suffering from serious $\mathrm{Li}$ dendrite penetration through grain boundaries ${ }^{16}$, pores ${ }^{17}$, and even through single crystals ${ }^{18}$.

It is generally acknowledged that interfacial properties play a critical role in regulating Li deposition ${ }^{19}$. LLZO shows poor wettability with Li metal. Large interfacial resistance which promotes Li dendrite nucleation is therefore difficult to prevent. Various strategies have been proposed to enhance the interfacial contact between LLZO and Li metal, such as introducing intermediate layers ${ }^{20-22}$, cleaning surface contaminants ${ }^{23,24}$, increasing the pressure or temperature ${ }^{25,26}$, and constructing a threedimensional (3D) interfacial structure ${ }^{27}$. These approaches improve the wettability and thus reduce Li dendrite propagation to some extent; however, lithium penetration through the electrolyte still occurs with increased current density or extended cycling time ${ }^{28}$. These results indicate that improvement of interfacial contact is not enough to address dendritic deposition on its own.

Although the mechanisms for Li dendrite growth remain elusive, the electron attack to the garnet electrolytes has recently received increased scrutiny since high electronic conductivity of SSEs was reported as the cause for certain types of Li dendrites ${ }^{29}$. The electron attack to the garnet electrolytes was recently visualized by a scanning electron microscope $(\mathrm{SEM})^{30}$. The electron beam in the SEM irradiated on the Ta-doped LLZO (LLZTO) surface can expulse the Li out of the LLZTO to satisfy the neutrality. Due to heterogeneous $\mathrm{Li}^{+}$transport in LLZTO electrolytes, $\mathrm{Li}^{+}$preferentially accumulates in defects and voids, forming metallic $\mathrm{Li}$ as it combines with electrons ${ }^{31,32}$. Poor interfacial contact leads to an uneven electric field, resulting in large local currents at the interface and promoting rapid $\mathrm{Li}$ dendrite penetration through the electrolyte. The construction of an electron-blocking interface with excellent wettability is therefore important for the development of dendrite-free Li anodes in $\mathrm{SSBs}^{33}$. Unfortunately, most interlayers alloy with Li metal and are electronically conductive, while ionically conductive but electronically insulating materials show large interfacial resistance with Li metal ${ }^{34}$. A hybrid interlayer formed with electronically conductive nanoparticles embedded in an ionically conductive matrix was recently reported to achieve excellent interfacial wettability and a uniform electric field distribution ${ }^{28}$; however, the improvement of electrochemical performance was still limited due to the conductive interface not preventing electron mobility within the garnet electrolyte.

Herein, we propose a flexible electron-blocking interfacial shield (EBS) to achieve uniform interfacial contact and prevent dendritic deposits attributed to high electronic conductivity in garnet electrolytes. Polyacrylic acid (PAA) polymer at the interface reacts with molten $\mathrm{Li}$ at $250^{\circ} \mathrm{C}$, forming Li-inserted PAA
(LiPAA). Such an EBS leads to good wettability with Li metal, decreasing the interfacial resistance from 1104.3 to $54.5 \Omega \mathrm{cm}^{2}$ at $25^{\circ} \mathrm{C}$. In addition, the flexible polymer interface relieves the interfacial stress generated by the changing volume of the $\mathrm{Li}$ anode, thus maintaining excellent interfacial contact during cycling ${ }^{35}$. The electron-blocking nature of the EBS is supported by density functional theory (DFT) calculations. Electrostatic potential profiles and density of states (DOS) profiles show that the LLZTO electrolyte and surface $\mathrm{Li}_{2} \mathrm{CO}_{3}$ contamination conduct electrons, while the EBS is electrically insulating. As a proof of concept, garnet electrolytes with EBS show improved performance in both $\mathrm{Li}$ symmetric cells and $\mathrm{LiFePO}_{4} / \mathrm{Li}$ cells.

\section{Results}

Characterizations of the LLZTO@PAA. LLZTO ceramic pellets were fabricated by the hot-press sintering technique detailed in our previous study ${ }^{36}$. Cross-sectional SEM images of LLZTO show a transgranular fracture morphology without obvious grain boundaries, leading to a high relative density of over $99.5 \%$ (Supplementary Fig. 1a) ${ }^{37}$. The X-ray diffraction (XRD) pattern, shown in Supplementary Fig. 2, shows diffraction peaks which match well with the standard pattern of cubic-phase garnet electrolytes (PDF\#45-0109). The high relative density and pure cubic phase result in an ionic conductivity as high as $1.1 \times$ $10^{-3} \mathrm{~S} \mathrm{~cm}^{-1}$ at $25^{\circ} \mathrm{C}$ (Supplementary Fig. 1b). PAA exhibits an amorphous structure with a broad peak at $2 \theta=\sim 18^{\circ 38}$. The PAA was dissolved in a dimethyl sulfoxide (DMSO) solution and coated on the surface of the LLZTO by drip casting. To evaluate the chemical stability between the LLZTO, PAA, and DMSO, the LLZTO particles were mixed with the PAA slurry and the DMSO solvent evaporated at $80^{\circ} \mathrm{C}$. The XRD pattern in Supplementary Fig. 2 shows no change to the garnet structure, confirming the stability of the constituent components.

Time-of-flight secondary-ion mass spectroscopy (TOF-SIMS) was carried out to examine the thickness and homogeneity of the PAA thin films on garnet electrolytes. TOF-SIMS depth profiling reveals the composition of fragments from the specimen during the sputtering process ${ }^{39}$. Here, $\mathrm{CHO}_{2}^{-}$and $\mathrm{C}_{2} \mathrm{HO}^{-}$fragments originate from the PAA layer, while $\mathrm{LaO}_{2}{ }^{-}, \mathrm{ZrO}_{2}{ }^{-}$, and $\mathrm{TaO}_{2}{ }^{-}$ fragments come from the LLZTO underneath. As shown in Fig. 1a, the $\mathrm{CHO}_{2}{ }^{-}$and $\mathrm{C}_{2} \mathrm{HO}^{-}$signal intensities are initially high, but gradually decline after $45 \mathrm{~s}$ of $\mathrm{Cs}^{+}$sputtering. In contrast, the $\mathrm{LaO}_{2}{ }^{-}, \mathrm{ZrO}_{2}{ }^{-}$, and $\mathrm{TaO}_{2}{ }^{-}$signals from the LLZTO initially weak, but gradually increase during the $45 \mathrm{~s}$ of $\mathrm{Cs}^{+}$ sputtering. A uniform PAA film is thus shown to coat the LLZTO pellet. The thickness of the PAA coating is estimated to be $43 \mathrm{~nm}$ based on a sputtering rate of $0.96 \mathrm{~nm} \mathrm{~s}^{-1}$. Supplementary Fig. 3 shows the TOF-SIMS mappings of the $\mathrm{CHO}_{2}{ }^{-}, \mathrm{C}_{2} \mathrm{HO}^{-}, \mathrm{LaO}_{2}{ }^{-}$, $\mathrm{ZrO}_{2}{ }^{-}$, and $\mathrm{TaO}_{2}{ }^{-}$signals after sputtering. Strong $\mathrm{LaO}_{2}{ }^{-}$, $\mathrm{ZrO}_{2}{ }^{-}$, and $\mathrm{TaO}_{2}{ }^{-}$signals corresponding to LLZTO are observed from the sputtered region and intense $\mathrm{CHO}_{2}^{-}$and $\mathrm{C}_{2} \mathrm{HO}^{-}$ signals corresponding to the PAA are observed across the pristine region. Three-dimensional views of the sputtered volume of LLZTO@PAA directly visualize the homogeneous coverage of PAA on the surface of the LLZTO electrolyte (Fig. 1b). In addition, Supplementary Fig. 4 shows the cross-sectional SEM image and energy dispersion spectrum (EDS) scanning of the PAA-coated LLZTO pellet. The thickness of the uniform PAA film is $\sim 48 \mathrm{~nm}$, which is consistent with the result of TOF-SIMS.

Topographical atomic force microscopy (AFM) images indicate that loose surface contaminants (e.g. $\mathrm{Li}_{2} \mathrm{CO}_{3}$ ) from exposure to air leave LLZTO pellets with a rough surface (Fig. 1c) ${ }^{40}$. The surface becomes relatively smooth after coating with the PAA film (Fig. 1e). Interfacial hardness can greatly affect Li dendrite growth due to residual stresses during repeated cycling ${ }^{41}$. 

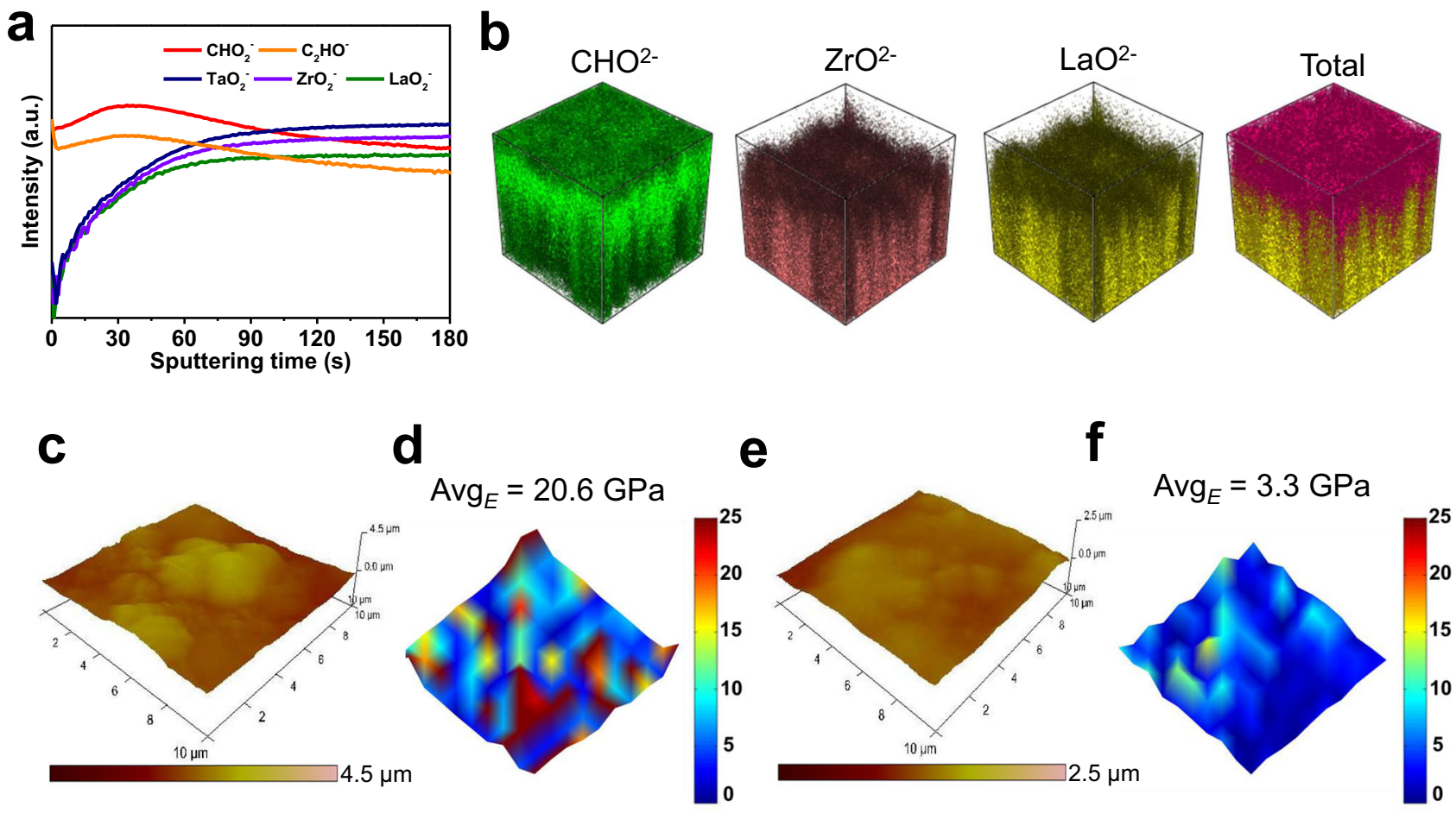

Fig. 1 Physical properties of the PAA layer on the surface of LLZTO. a TOF-SIMS depth profiles for the LLZTO@PAA pellet. $\mathbf{b} 3 D$ views of the sputtered volume of LLZTO@PAA. c AFM topography image, and $\mathbf{d}$ Young's modulus of LLZTO. e AFM topography image, and $\mathbf{f}$ Young's modulus of LLZTO@PAA.

Interfaces with poor ductility may be broken by the Li volume change, leading to poor interfacial contact and large resistance ${ }^{35}$. A soft interface is therefore required to relieve interfacial stress and maintain good interfacial contact. To compare the surface hardness before and after coverage with PAA, Young's modulus (E) mappings were created by fitting force-distance curves at 100 locations in a $30 \times 30 \mu \mathrm{m}^{2}$ area. The average Young's modulus $\left(\operatorname{Avg}_{E}\right)$ for LLZTO is $20.6 \mathrm{GPa}$, while the $\mathrm{Avg}_{E}$ for LLZTO@PAA is $3.3 \mathrm{GPa}$ (Fig. 1d and $\mathrm{f}$ ). The decreased $\mathrm{Avg}_{E}$ indicates a flexible interface which can serve as a stable interface during cycling and suppress Li dendrite growth.

Formation of the EBS by the substitution reaction. The EBS was formed in situ by the reaction of a PAA film with molten Li at $250^{\circ} \mathrm{C}$. The reaction mechanisms and products were investigated using first-principles calculations. Supplementary Fig. 5a and $b$ shows there are two possible reaction mechanisms between PAA and molten Li. One is a recombination reaction, where $\mathrm{Li}$ inserts directly into PAA polymer chains. The other is a substitution reaction, where $\mathrm{Li}$ replaces the $\mathrm{H}$ in a $\mathrm{PAA}-\mathrm{COOH}$ group. The electrostatic potential profiles in Supplementary Fig. $5 \mathrm{c}$ show that the dehydrogenated interphase created by the substitution reaction is more stable. Differential electrochemical mass spectrometry (DEMS) was used to detect the $\mathrm{H}_{2}$ release and further confirm the substitution reaction (Supplementary Fig. 6 and Supplementary Note 1). The escaping electrons accompanied by $\mathrm{H}_{2}$ gas release suppress the interfacial electrostatic potential and prohibit electron permeation. The structure and composition of LiPAA were studied by SEM, X-ray photoelectron spectroscopy (XPS), Fourier Transform Infrared Spectroscopy (FTIR), and Raman, which can support the results of theoretical calculations (Supplementary Fig. 7 and Supplementary Note 2).

The work of adhesion $\left(W_{\text {ad }}\right)$ for dehydrogenated PAA on Li metal is $60.1 \mathrm{meV}^{-2}$, much higher than the $58.0 \mathrm{meV}^{-2}$ for LLZTO $(110) / \mathrm{Li}(001)$ and the $16.5 \mathrm{meV}^{-2}$ for $\mathrm{Li}_{2} \mathrm{CO}_{3}(001) / \mathrm{Li}(001)$
(Fig. 2a, b and Supplementary Fig. 8a). Note that $\mathrm{Li}_{2} \mathrm{CO}_{3}$ is the main component of the contamination on LLZTO surfaces exposed to air ${ }^{40}$. As a result, the PAA layer improves the wettability of Li metal on LLZTO, especially when the LLZTO is covered by lithiophobic $\mathrm{Li}_{2} \mathrm{CO}_{3}$. The contact angle was calculated with the following equation:

$$
W_{\mathrm{ad}}=\sigma_{\mathrm{Li}}(1+\cos \theta)=\frac{E_{\text {interface }}-E_{\mathrm{Li}-\text { slab }}-E_{\mathrm{PAA}\left(\mathrm{LLZTO} / \mathrm{Li}_{2} \mathrm{CO}_{3}\right)-\text { slab }}}{S},
$$

where $W_{\mathrm{ad}}$ is the interfacial work of adhesion, $\sigma_{\mathrm{Li}}$ is the surface energy of $\mathrm{Li}$, and $\theta$ is the contact angle ${ }^{42,43}$. The $\theta$ for PAA/Li, $\mathrm{LLZTO} / \mathrm{Li}$, and $\mathrm{Li}_{2} \mathrm{CO}_{3} / \mathrm{Li}$ is $\sim 0^{\circ}, 85.9^{\circ}$, and $132.7^{\circ}$, respectively, indicating greatly improved wettability between the LLZTO and Li metal using a PAA intermediate layer.

Electron-blocking property of the EBS. A LiPAA EBS effectively blocks electrons at the interface. This fact is confirmed by the electrostatic potential profiles and DOS simulation results shown in Fig. 2c and Supplementary Fig. 8b for LLZTO(110)/Li(001) and $\mathrm{Li}_{2} \mathrm{CO}_{3}(001) / \mathrm{Li}(001)$, respectively. There is no barrier to the transfer of electrons from the interface to the LLZTO electrolyte. In the case of $\operatorname{LLZTO}(110) / \mathrm{Li}(001)$, electrons and $\mathrm{Li}$ atoms preferentially deposit within the LLZTO rather than at the LLZTO/Li interface, a behavior corroborated by DOS results. The result is that LLZTO becomes electronically conductive when lithiated (Fig. 2d and e), forming Li dendrites across LLZTO electrolytes ${ }^{29}$. The interfacial electron density of $\mathrm{Li}_{2} \mathrm{CO}_{3}(001) / \mathrm{Li}(001)$ is higher than that of $\operatorname{LLZTO}(110) / \mathrm{Li}(001)$. An abnormal space charge layer is shown in Supplementary Fig. $8 c$ and d. The outer layer has a slightly higher electronic DOS than the inner layer, indicating that insulative $\mathrm{Li}_{2} \mathrm{CO}_{3}$ promotes electron permeation due to complex interfacial phenomena. In contrast, the electrostatic potential of the PAA/Li(001) interface is $1.92 \mathrm{eV}$ lower than LiPAA polymer, which is attributed to the dehydrogenation reaction (Fig. 2f). 

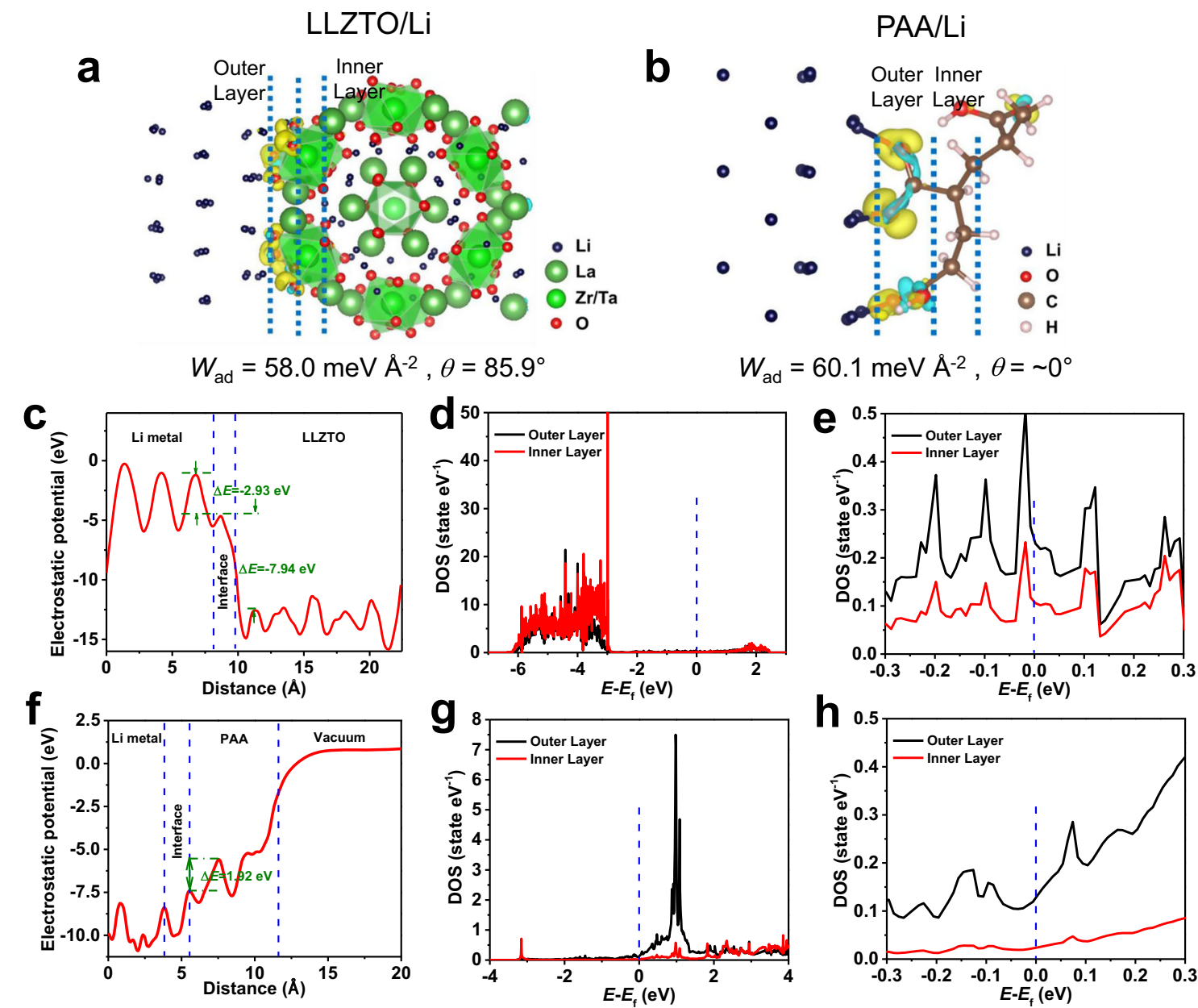

Fig. 2 Electron-blocking property of the PAA-modified LLZTO with Li metal. a The structure and charge transfer, $\mathbf{c}$ the electrostatic potential profiles, and d, e the density of states (DOS) for fully relaxed LLZTO(110)/Li(001). $\mathbf{b}$ The structure and charge transfer, $\mathbf{f}$ the electrostatic potential profiles, and $\mathbf{g}$, $\mathbf{h}$ the density of states (DOS) for fully relaxed PAA/Li(001) single chain (the vacuum slab is not entirely presented in the model).

Electrons are contained to the Li metal and permeate only into the outer layer of the interface. In addition, $\mathrm{Li}$ deposition occurs preferentially at the interface rather than within the LiPAA, prohibiting the penetration of $\mathrm{Li}$ dendrites through the PAA. The electronically insulating nature is further confirmed by DOS results for PAA/Li(001), shown in Fig. $2 \mathrm{~g}$ and h. Electrons are captured within the Li/PAA interfacial bonds, while the inner layer remains insulating. To further confirm the electronically insulating property of LiPAA, the electronic conductivity of the LLZTO and the LLZTO@EBS was evaluated by DC polarization at $0.1 \mathrm{~V}$. As shown in Supplementary Fig. 9, the electronic conductivity of the LLZTO@EBS is smaller than that of the LLZTO, indicating the excellent capability of electron block by the EBS.

The LLZTO@EBS/Li wettability was evaluated with molten Li and LLZTO@PAA. As shown in Fig. 3a, molten Li forms a sphere on the LLZTO surface, indicating a large $\theta$. This poor wettability leads to gaps at the interface. In contrast, molten Li completely wets LLZTO@EBS (Fig. 3b). Cross-sectional SEM image shows intimate contact between the LLZTO@EBS and the Li metal without any voids at the interface. This enhanced wettability is consistent with the simulated $\theta$. Complete wetting significantly decreases the interfacial resistance, thus improving electrochemical performance.

SSBs benefitting from the EBS. Li/LLZTO@EBS/Li and $\mathrm{Li} / \mathrm{LLZTO} / \mathrm{Li}$ symmetric cells were assembled for electrochemical characterization. Electrochemical impedance spectroscopy (EIS) was carried out to compare the interfacial resistance of cells with and without the EBS. Figure $3 \mathrm{c}$ shows the impedance spectra obtained at $25^{\circ} \mathrm{C}$. The impedance spectrum of the Li/LLZTO/Li cell exhibits one large semicircle. The starting point of the spectrum corresponds to the bulk resistance of the LLZTO, while the semicircle corresponds to the interfacial resistance between LLZTO and Li metal ${ }^{37}$. In an ideal situation, the charge transfer across two Li/LLZTO interfaces should be identical in a symmetric cell. The interfacial resistance determined from the semicircle is divided by two to obtain the value for each Li/LLZTO interface. Thus, the LLZTO/Li interfacial resistance is found to be $1104.3 \Omega \mathrm{cm}^{2}$. The Li/LLZTO@EBS/Li symmetric cell shows multiple semicircles resulting from the EBS bulk and EBS/ LLZTO interface at high frequency and EBS/Li interface at low frequency. The overall resistance of the LLZTO@EBS/Li interface was $54.5 \Omega \mathrm{cm}^{2}$. The decrease in interfacial resistance from $1104.3 \Omega \mathrm{cm}^{2}$ to $54.5 \Omega \mathrm{cm}^{2}$ can be ascribed to the lithiophilicity of the EBS film. In addition, the temperature-dependence of the interfacial resistance was characterized between $25^{\circ} \mathrm{C}$ and $85^{\circ} \mathrm{C}$. The activation energy $\left(E_{\mathrm{a}}\right)$ of the EBS modified and the unmodified interface was calculated using the Arrhenius law. The $E_{\mathrm{a}}$ of the LLZTO@EBS/Li interface is $0.38 \mathrm{eV}$, while the $E_{\mathrm{a}}$ of the LLZTO / Li interface is $0.51 \mathrm{eV}$ (Fig. 3d). The decreased $E_{\mathrm{a}}$ is beneficial for $\mathrm{Li}^{+}$migration across the interface ${ }^{28}$.

The critical current density (CCD) was used as a measure of the interfacial stability and capacity for Li dendrite suppression. 
a

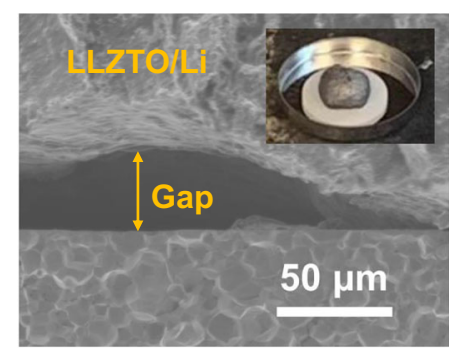

b

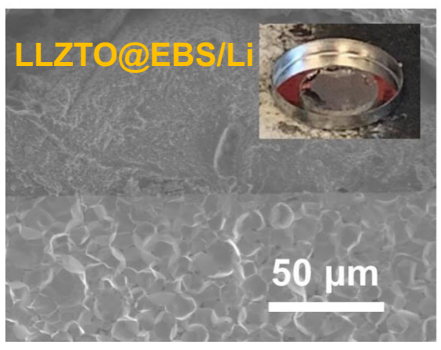

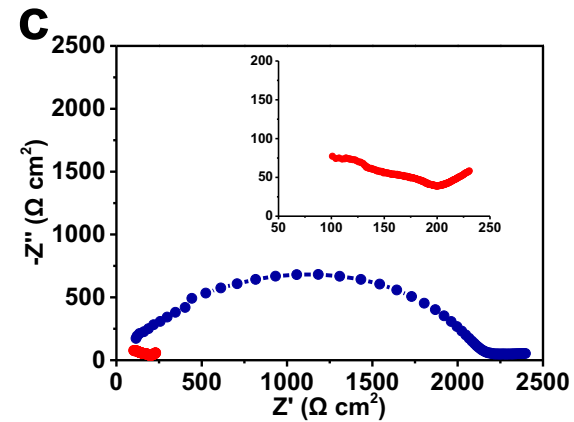

e

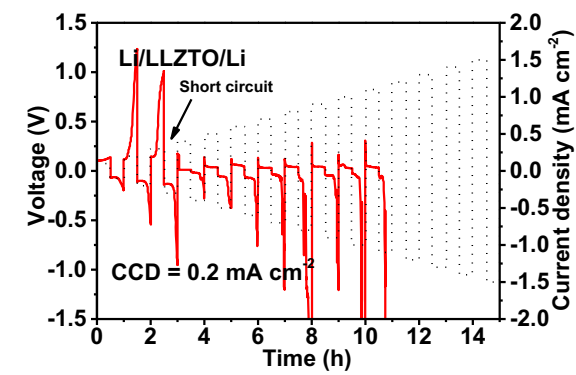

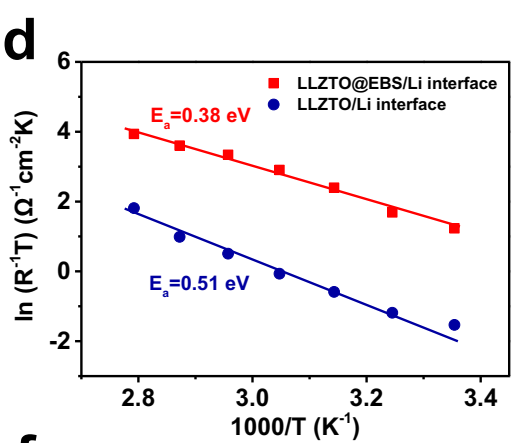

$f$

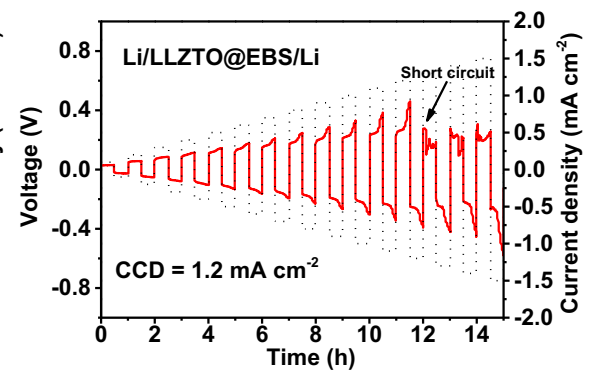

Fig. 3 Comparation of interfacial wetting behaviors between bare LLZTO and PAA-modified LLZTO with molten Li. SEM images of a the LLZTO/Li interface and $\mathbf{b}$ the LLZTO@EBS/Li interface. Insets are the corresponding digital images showing the wetting behaviors of molten Li on bare LLZTO or LLZTO@EBS. c EIS spectra, d temperature-dependent interfacial resistance of the Li/LLZTO/Li and the Li/LLZTO@EBS/Li cells. e CCD of the Li/LLZTO/Li cell. $\mathbf{f}$ CCD of the Li/LLZTO@EBS/Li cell.

The CCD is defined as the current density where the cell reaches a short circuit. An applied current density was increased from 0.1 to $1.5 \mathrm{~mA} \mathrm{~cm}^{-2}$ with a step increase of $0.1 \mathrm{~mA} \mathrm{~cm}^{-2}$ per hour at $25^{\circ} \mathrm{C}$. Figure $3 \mathrm{e}$ shows that the CCD of the $\mathrm{Li} / \mathrm{LLZTO} / \mathrm{Li}$ cell is as low as $0.2 \mathrm{~mA} \mathrm{~cm}^{-2}$. The large overpotential over $1 \mathrm{~V}$ is a result of poor interfacial contact. The CCD of the Li/LLZTO@EBS/Li cell is significantly improved to $1.2 \mathrm{~mA} \mathrm{~cm}^{-2}$. The voltage profile of the Li/LLZTO@EBS/Li cell remains relatively stable before short circuiting. The improvement in CCD can be attributed to combined contributions from the electronically insulating interface and from the relieved interfacial stress. More specifically, the LiPAA EBS facilitates $\mathrm{Li}^{+}$transport and prevents electronic degradation of the LLZTO bulk. In addition, the flexibility of the polymer interface alleviates interfacial stress, maintaining interfacial contact and suppressing $\mathrm{Li}$ dendrite growth. To our knowledge, a CCD of $1.2 \mathrm{~mA} \mathrm{~cm}^{-2}$ at room temperature is the highest value ever reported for garnet electrolytes (Supplementary Table 1). Despite the various surface modification approaches used to decrease interfacial resistance by enhancing wettability, the CCD is still limited due to electronic degradation and poor interfacial stability at high current densities.

Galvanostatic Li plating/stripping experiments were carried out to evaluate the long-term stability of $\mathrm{Li}^{+}$transport and the effectiveness of dendrite suppression at the interface. As shown in Fig. 4a, the Li/ LLZTO/Li cell exhibits an overpotential over $0.45 \mathrm{~V}$ for the first charge/discharge cycle at $0.2 \mathrm{~mA} \mathrm{~cm}^{-2}\left(0.1 \mathrm{mAh} \mathrm{cm}^{-2}\right)$, indicating inhomogeneous $\mathrm{Li}$ deposition. A short circuit occurs within three cycles. The poor LLZTO/Li interfacial contact leads to uneven current distribution and serious electronic degradation at the defects, thus inducing Li dendrite growth ${ }^{44}$. In contrast, the Li/LLZTO@EBS/ $\mathrm{Li}$ cell continuously operates for over $1000 \mathrm{~h}$ with an overpotential of $46.1 \mathrm{mV}$ at $0.2 \mathrm{~mA} \mathrm{~cm}^{-2}$ (Fig. 4b). Moreover, the Li/LLZTO@EBS/Li cell shows stable cycling for $400 \mathrm{~h}$ at $0.5 \mathrm{~mA} \mathrm{~cm}^{-2}\left(0.25 \mathrm{mAh} \mathrm{cm}^{-2}\right)$, while the $\mathrm{Li} / \mathrm{LLZTO} / \mathrm{Li}$ cell cannot be cycled even once (Fig. $4 \mathrm{c}$ and d). Increasing the current density and areal capacity to $1 \mathrm{~mA} \mathrm{~cm}-2$ and $1 \mathrm{mAh} \mathrm{cm}^{-2}$, the Li/LLZTO-EBS/Li cell continues to show stable cycling for $400 \mathrm{~h}$ (Fig. 4e). To our knowledge, the performance of LLZTO@EBS is superior to the performance achieved with garnet electrolytes in all previous studies (Fig. 4f).

After disassembling the short-circuited Li/LLZTO/Li cell and reacting the $\mathrm{Li}$ metal with a water/alcohol solution, a rough LLZTO surface with voids and defects is revealed. The dark spots reveal areas where Li dendrites have grown into the LLZTO pellet (Fig. 5a). This is confirmed by SEM (Fig. 5b). The cross-sectional SEM image shows the proliferation of $\mathrm{Li}$ dendrites through the LLZTO grain boundaries (Fig. $5 \mathrm{c}$ and $\mathrm{d}$ ), the cause of short circuiting. As shown in Fig. $5 \mathrm{e}$ and $\mathrm{f}$, the surface of the LiPAAprotected LLZTO remains smooth after $1000 \mathrm{~h}$ without dark spots from dendrites. The flexible polymer EBS accommodates the $\mathrm{Li}$ volume change to maintain good contact (Fig. $5 \mathrm{~g}$ ). The slight increase in overall resistance from 209.1 to $224.3 \Omega \mathrm{cm}^{2}$ confirms that no short circuiting occurs after $1000 \mathrm{~h}$ of cycling (Supplementary Fig. 10). The dendrite-free grain boundary of the LLZTO further confirms the ability of the EBS to prevent dendrite growth (Fig. 5h).

\section{Discussion}

To understand the mechanisms for dendrite suppression by the EBS, other interfacial layers were synthesized between the LLZTO electrolyte and Li metal for comparison. A Au layer was first coated on the LLZTO surface by sputtering. Supplementary Fig. 11a shows the excellent wettability of the LLZTO@Au with molten $\mathrm{Li}$ due to the formation of a $\mathrm{Au}-\mathrm{Li}$ alloy at $300^{\circ} \mathrm{C}$. This enhanced wettability leads to a dramatically decreased interfacial resistance of $43.2 \Omega \mathrm{cm}^{2}$ (Supplementary Fig. 11b). The Li/ LLZTO@Au/Li cell exhibits a CCD of $0.7 \mathrm{~mA} \mathrm{~cm}^{-2}$ and stable cycling over $200 \mathrm{~h}$ at $0.5 \mathrm{~mA} \mathrm{~cm}^{-2}\left(0.25 \mathrm{mAh} \mathrm{cm}^{-2}\right)$. This is a marked improvement over the pristine Li/LLZTO/Li cell (Supplementary Figs. 11c and 12) where poor wettability of the LLZTO causes an uneven electric field and local hot spots which lead to Li dendrite nucleation and propagation (Fig. 6a). The 

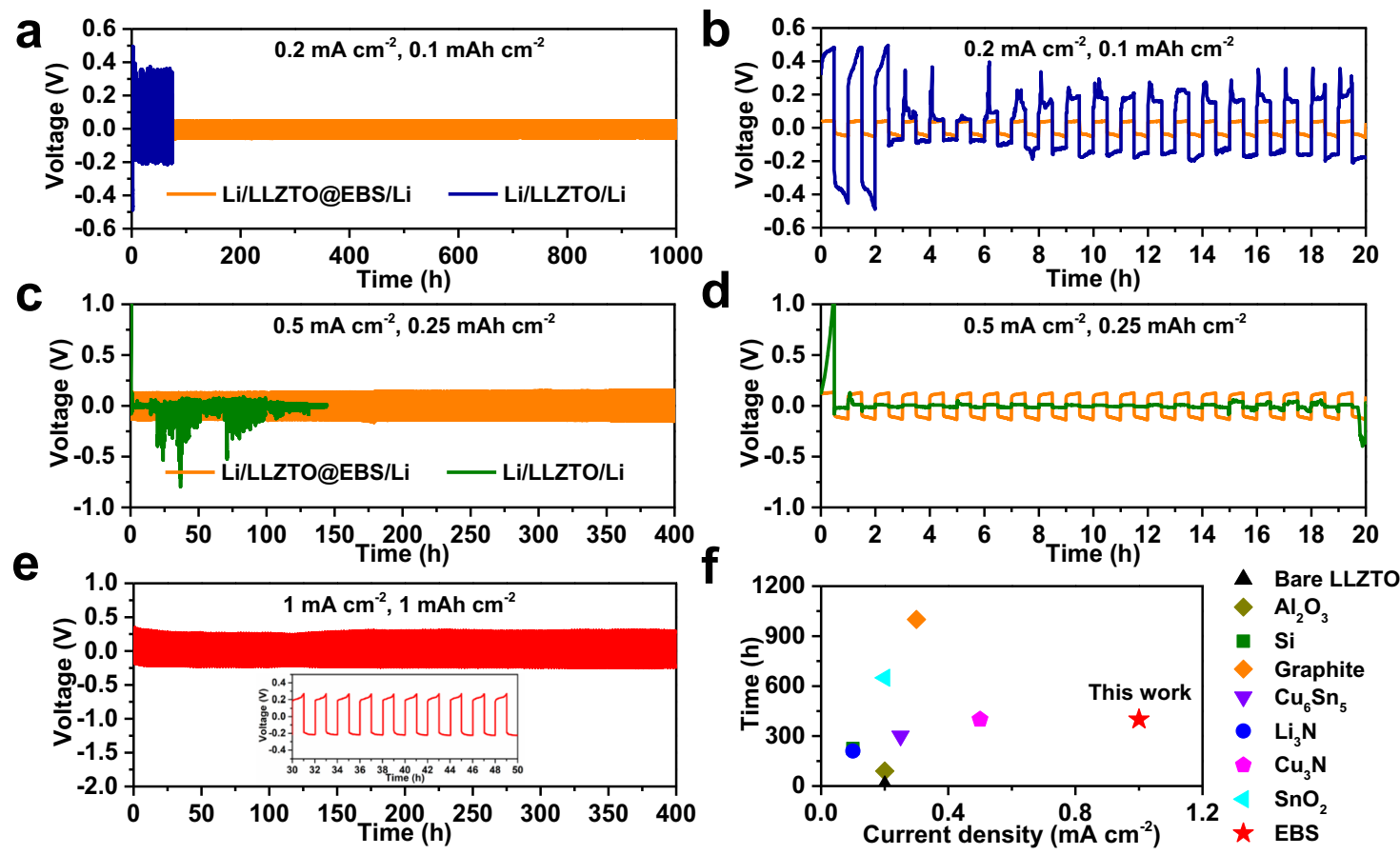

Fig. 4 Electrochemical performance of Li symmetric cells using bare LLZTO and the PAA-modified LLZTO. a Galvanostatic cycling performance of the Li/LLZTO/Li and Li/LLZTO@EBS/Li cells at $0.2 \mathrm{~mA} \mathrm{~cm}^{-2}\left(0.1 \mathrm{mAh} \mathrm{cm}^{-2}\right)$ at $25^{\circ} \mathrm{C}$; b Magnified images for 0-20 h. c Galvanostatic cycling performance of the Li/LLZTO/Li and Li/LLZTO@EBS/Li cells at $0.5 \mathrm{~mA} \mathrm{~cm}^{-2}\left(0.25 \mathrm{mAh} \mathrm{cm}^{-2}\right)$ at $25^{\circ} \mathrm{C}$; d Magnified images for $0-20 \mathrm{~h}$. e Galvanostatic cycling performance of the Li/LLZTO@EBS/Li cell at $1 \mathrm{~mA} \mathrm{~cm}-2\left(1 \mathrm{mAh} \mathrm{cm}{ }^{-2}\right)$ at $25^{\circ} \mathrm{C}$. $\mathbf{f}$ Galvanostatic cycling performance in recent literature compared with our work $20,21,28,35,37,58,59$.

a

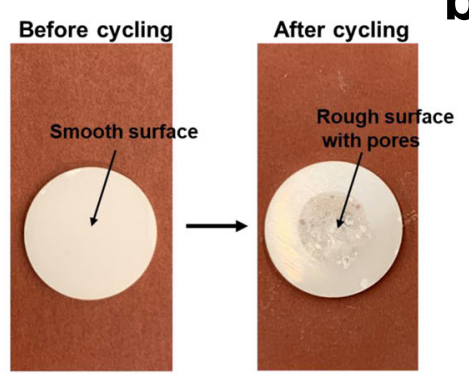

e

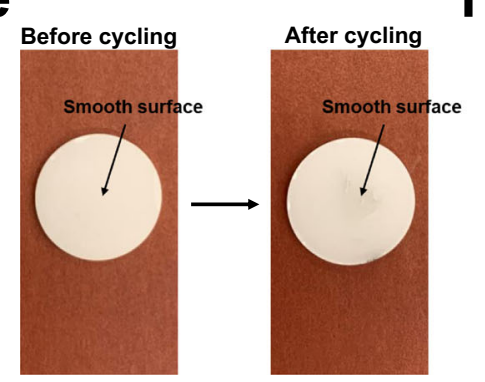

b

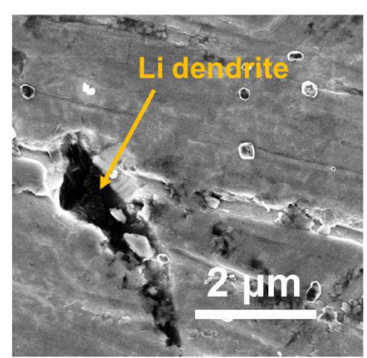

$\mathbf{f}$

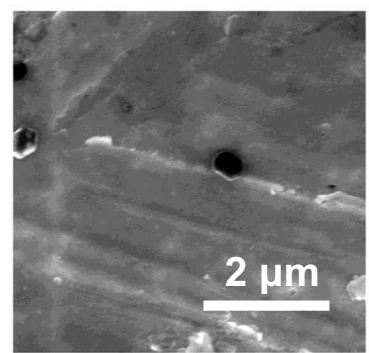

C

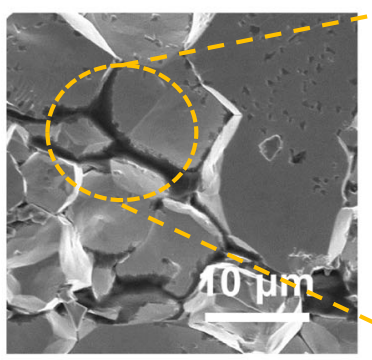

g

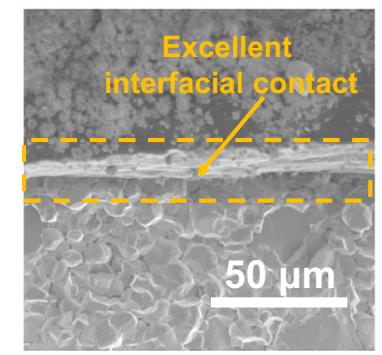

d

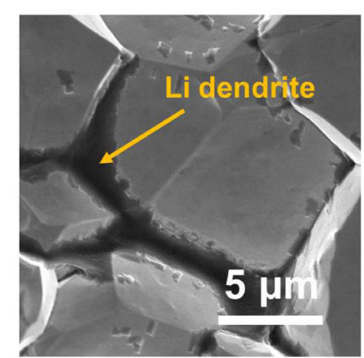

h

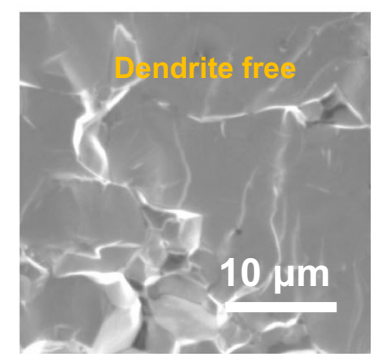

Fig. 5 Li dendrite growth in the bare LLZTO and the PAA-modified LLZTO. a Optical surface morphologies of an LLZTO pellet before and after cycling. b Top-view and c, d cross-sectional SEM images of an LLZTO pellet after short circuiting. e Optical surface morphologies of an LLZTO@EBS pellet before and after cycling. f Top-view SEM image of an LLZTO@EBS pellet after cycling at $0.2 \mathrm{~mA} \mathrm{~cm}^{-2}$ for $1000 \mathrm{~h}$. $\mathbf{g}$ Cross-sectional SEM image of the LLZTO@EBS/Li interface, and $\mathbf{h}$ cross-sectional SEM image of an LLZTO@EBS pellet after cycling at $0.2 \mathrm{~mA} \mathrm{~cm}^{-2}$ for $1000 \mathrm{~h}$.

improved cycling performance achieved with a $\mathrm{Au}$ interfacial provides a basis for the idea that interfacial wettability is important for the uniform distribution of the electric field (Fig. 6b).
Although the interfacial resistance of LLZTO@Au/Li is smaller than that of LLZTO@EBS/Li, the Li/LLZTO@Au/Li cell shows a much shorter cycle life than Li/LLZTO@EBS/Li cell at $0.5 \mathrm{~mA} \mathrm{~cm}^{-2}$ (Supplementary Fig. 12). This can be attributed to the electron 

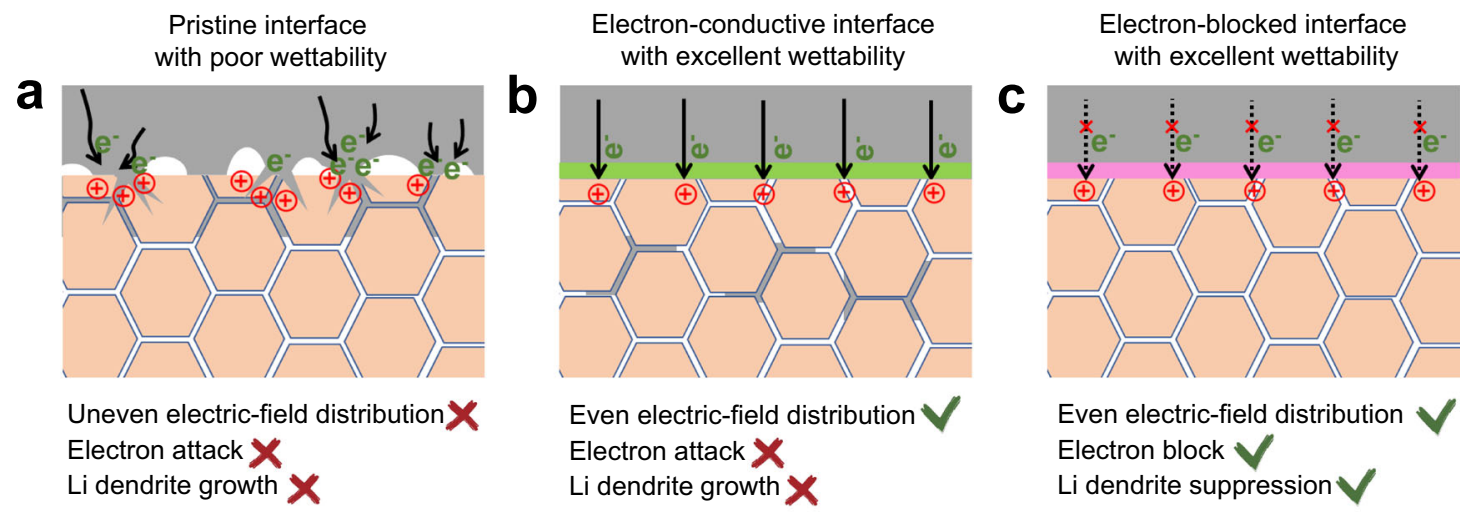

\begin{abstract}
Li metal Au-Li alloy LiPAA

$\oplus \mathrm{Li}^{+} \quad \mathrm{e}^{-}$Electron

Electron transport $\cdots . . . . \vee$ Electron block

Fig. 6 Schematic illustrations of Li dendrite growth at various interfaces. Schematic illustrations of Li dendrite growth at the a pristine LLZTO/Li interface, b LLZTO@Au/Li interface, and c LLZTO@EBS/Li interface.
\end{abstract}

$\operatorname{attack}^{29}$. Electrons within the electrolyte can combine with lithium ions to form $\mathrm{Li}$ metal within the polycrystalline electrolytes, especially at the grain boundaries. Pristine LLZTO with its lithiophobic nature accumulates electrons unevenly at locations with point contact, more readily forming $\mathrm{Li}$ dendrites (Fig. 6a). The LLZTO@Au is also unable to avoid this form of degradation due to the conductive nature of the interface (Fig. 6b). In contrast, the EBS protected LLZTO prevents electrons from entering the electrolyte, avoiding dendrite formation (Fig. 6c) ${ }^{45}$. The flexible LiPAA polymer maintains interfacial contact by accommodating the Li volume change during cycling. In contrast, the overpotential of the Li/ LLZTO@Au/Li cell gradually increases after $150 \mathrm{~h}$ cycling due to fracturing of the interface, accelerating the short circuit (Supplementary Fig. 12).

To further explore the capability of the EBS layer in suppressing dendrites, PAA was coated on LLZTO electrolytes with a relative density of $96 \%$ (LLZTO(96\%)). LLZTO(96\%) facilitates Li dendrite growth compared to high-density LLZTO. The crosssectional SEM image, shown in Supplementary Fig. 13a, shows that the LLZTO(96\%) consists of small garnet grains with many grain boundaries and voids, leading to a decreased ionic conductivity of $5.4 \times 10^{-4} \mathrm{~S} \mathrm{~cm}^{-1}$ at $25^{\circ} \mathrm{C}$ (Supplementary Fig. 13b). The increased number of grain boundaries trap electrons and lead to increased $\mathrm{Li}$ dendrite formation ${ }^{32,46}$. Supplementary Fig. 14 shows short-circuiting of the Li/LLZTO(96\%)@Au/Li cell after $18 \mathrm{~h}$ of cycling at $0.2 \mathrm{~mA} \mathrm{~cm}^{-2}\left(0.1 \mathrm{~mA} \mathrm{~cm}^{-2}\right)$. SEM images show a large amount of mossy Li dendrite within the grain boundaries in the short-circuited LLZTO(96\%) electrolyte (Supplementary Fig. 15). In contrast, the Li/LLZTO(96\%)@EBS/ Li cell demonstrates stable cycling for $150 \mathrm{~h}$ at $0.2 \mathrm{~mA} \mathrm{~cm}^{-2}$ (Supplementary Fig. 14).

$\mathrm{Li}^{+}$transport at the interface also affects $\mathrm{Li}$ dendrite growth. For comparison, a traditional poly(ethylene oxide)/Lithium bis(trifluoromethanesulfonyl)imide electrolyte layer (PEO) was coated on the surface of LLZTO pellets. Supplementary Fig. 16a shows a PEO layer thickness of $\sim 5 \mu \mathrm{m}$. The EIS spectrum for the Li/LLZTO@$\mathrm{PEO} / \mathrm{Li}$ cell shows two semicircles at $60^{\circ} \mathrm{C}$ (Supplementary Fig. 16b). The small semicircle at a high frequency corresponds to the resistance of the PEO layer, while the large semicircle at a low frequency is attributed to the resistance of the LLZTO/PEO and $\mathrm{PEO} / \mathrm{Li}$ interfaces. The interfacial resistance resulting from the $\mathrm{PEO}$ modification is $683.2 \Omega \mathrm{cm}^{2}$ at $60^{\circ} \mathrm{C}$, one order of magnitude larger than the interfacial resistance of the EBS modification. Although the PEO layer is also electronically insulating, the sluggish $\mathrm{Li}^{+}$ transport at the interface leads to large overpotentials during the
CCD test. The CCD of the $\mathrm{Li} / \mathrm{LLZTO} / \mathrm{PEO} / \mathrm{Li}$ cell is $0.6 \mathrm{~mA} \mathrm{~cm}^{-2}$ (Supplementary Fig. 16c). In addition, polymer electrolytes with a $\mathrm{Li}$ salt as an intermediate layer always exhibit a low $\mathrm{Li}^{+}$transference number $(<0.5)$, which can induce uneven $\mathrm{Li}$ deposition 47,48 . In contrast, LiPAA polymer guides homogenous $\mathrm{Li}$ deposition without the interference of anions. The Li/LLZTO@PEO/Li cell shows an overpotential over $0.8 \mathrm{~V}$ and short circuits after $50 \mathrm{~h}$ of cycling at $0.5 \mathrm{~mA} \mathrm{~cm}^{-2}\left(0.25 \mathrm{mAh} \mathrm{cm}^{-2}\right)$ at $60{ }^{\circ} \mathrm{C}$ (Supplementary Fig. 17). The Li/LLZTO@EBS/Li cell operates continuously for $400 \mathrm{~h}$ at $0.5 \mathrm{~mA} \mathrm{~cm}^{-2}\left(0.25 \mathrm{mAh} \mathrm{cm}^{-2}\right)$ at $25^{\circ} \mathrm{C}$, indicating that the good $\mathrm{Li}^{+}$transport at the EBS interface is beneficial to performance.

To extend the substitution reaction to other polymers, PEO film was coated on the surface of the LLZTO pellets and reacted with the molten Li by the same method. The interfacial resistance of the $\mathrm{Li} / \mathrm{LLZTO}(\mathrm{PEO}) / \mathrm{Li}$ cell is even one order magnitude larger than that of the Li/LLZTO/Li cell without modification (Supplementary Fig. 18). The blocked $\mathrm{Li}^{+}$transport could be attributed to the following reasons: (1) compared with the $-\mathrm{OH}$ in the $\mathrm{PEO},-\mathrm{COOH}$ of the PAA as an acid group is easier to react with the Li by the substitution reaction; (2) the $-\mathrm{COOH}$ group of the $\mathrm{PAA}$ is on the main polymer chain, while the $-\mathrm{OH}$ group of the $\mathrm{PEO}$ is the terminal group. This leads to a larger number of $-\mathrm{COOH}$ of the PAA for the substitution reaction and the $\mathrm{Li}^{+}$ transportation by the segment movement; (3) PAA $\left(M_{\mathrm{w}}\right.$ : $\sim 450,000)$ shows the much smaller molecular weight than PEO $\left(M_{\mathrm{w}}: \sim 1,000,000\right)$, which may be beneficial for the $\mathrm{Li}^{+}$transport due to the decreased crystallinity.

Full SSBs with a $\mathrm{LiFePO}_{4}$ (LFP) cathode and a $\mathrm{Li}$ metal anode were constructed using LLZTO@EBS and compared to bare LLZTO. Supplementary Fig. 19a shows the configuration of the SSBs. The introduction of an ionic liquid as a wetting agent enhances $\mathrm{Li}^{+}$migration into the composite cathode for roomtemperature feasibility ${ }^{23,40}$. The LFP/LLZTO@EBS/Li cell shows smaller polarization than the LFP/LLZTO/Li cell at various current rates (Supplementary Fig. $19 \mathrm{~b}$ and c). The LFP/ LLZTO@EBS/Li cell delivers a specific discharge capacity of $142.3 \mathrm{mAh} \mathrm{g}^{-1}$ at $0.1 \mathrm{C}$. The discharge capacity is $130.2,119.5$, and $95.4 \mathrm{mAh} \mathrm{g}^{-1}$ at $0.2,0.5$, and $1 \mathrm{C}$, respectively (Supplementary Fig. 19d). After high-rate cycling, the cell can recover a discharge capacity of $142.5 \mathrm{mAh} \mathrm{g}^{-1}$ at $0.1 \mathrm{C}$. The high capacity and excellent cycling stability can be ascribed to good interfacial contact, an electronically insulating interface, and accommodation of the Li volume change. In contrast, the LFP/LLZTO/Li cell delivers a discharge capacity of $125.4 \mathrm{mAhg}^{-1}$ with a high 
overpotential at $0.1 \mathrm{C}$. The discharge capacity decreases to 105.2, 79.6, and $49.2 \mathrm{mAh} \mathrm{g}^{-1}$ at $0.2,0.5$, and $1 \mathrm{C}$, respectively (Supplementary Fig. 19d). Moreover, the SSB with LLZTO@EBS retains $82.8 \%$ capacity after 300 cycles at $0.2 \mathrm{C}$, and $83.1 \%$ capacity after 200 cycles at $0.5 \mathrm{C}$ at room temperature (Supplementary Fig. 19e and f). This excellent cycling performance is better than LFP/LLZTO/Li, LFP/LLZTO@Au/Li, and LFP/ LLZTO@PEO/Li cells (Supplementary Fig. 20).

In summary, a flexible LiPAA EBS is formed between an LLZTO electrolyte and Li metal anode to suppress Li dendrite growth. Interfacial resistance is dramatically decreased from 1104.3 to $54.5 \Omega \mathrm{cm}^{2}$ at $25^{\circ} \mathrm{C}$ due to a substitution reaction at the interface. The flexible EBS interface alleviates interfacial stress to maintain interfacial contact during cycling. The electronically insulating nature of the EBS is supported by electrostatic potential profiles and DOS results based on DFT simulations. EBSprotected LLZTO electrolytes prevent electronic degradation, avoiding the direct reduction of $\mathrm{Li}^{+}$to $\mathrm{Li}$ metal dendrites within LLZTO. Li/LLZTO@EBS/Li cells exhibit a CCD as high as 1.2 $\mathrm{mA} \mathrm{cm}{ }^{-2}$ at $25^{\circ} \mathrm{C}$. Li/LLZTO@EBS/Li cells can continuously operate for over $1000 \mathrm{~h}$ at $0.2 \mathrm{~mA} \mathrm{~cm}^{-2}$ and $400 \mathrm{~h}$ at $1 \mathrm{~mA} \mathrm{~cm}^{-2}$. The performance of an EBS layer is superior to electronconducting $\mathrm{Au}$ and traditional PEO polymer interfacial layers. This work represents the rational design of an interface for SSEs and Li metal anodes, and presents a promising strategy to achieve long-life and dendrite-free SSBs with high energy density and excellent safety.

\section{Methods \\ Fabrication of LLZTO@PAA. Ta-doped garnet $\mathrm{Li}_{6.4} \mathrm{La}_{3} \mathrm{Zr}_{1.4} \mathrm{Ta}_{0.6} \mathrm{O}_{12}$ (LLZTO)} powders were fabricated by the solid-state reactions, while LLZTO pellets were sintered by the hot-pressing technique ${ }^{36}$. The LLZTO ceramic pellets show the high relative density of $99.5 \pm 0.5 \%$, which was evaluated by the Archimedes' principle (Supplementary Table 2 and Supplementary Note 3). PAA (average $M_{\mathrm{w}} \sim 450,000$ ) polymer was dissolved in the DMSO solution by heating at $60^{\circ} \mathrm{C}$ for $12 \mathrm{~h}$. LLZTO@PAA was acquired by drip-casting the solution (0.25\% PAA in DMSO) on the surface of LLZTO pellets and then vacuum drying at $80^{\circ} \mathrm{C}$.

Material characterization. Crystal structures of samples were examined by XRD (Bruker D2 Phaser), using $\mathrm{Cu} \mathrm{Ka}$ radiation with $2 \theta$ in the range of $10^{\circ}-80^{\circ}$ and a step size of $0.02^{\circ}$. Surface and cross-section morphologies of the LLZTO pellets were investigated by scanning electron microscopy (SEM, S3400). TOF-SIMS testing was conducted using TOF-SIMS IV (ION-TOF GmbH, Germany) with a $25 \mathrm{keV}$ bismuth liquid metal ion source and a base pressure of $\approx 10^{-8}$ mbar in the analysis chamber. Negative secondary ions were induced by primary ion beam bombardment on the surface of LLZTO. The analysis area was $334 \mu \mathrm{m} \times 334 \mu \mathrm{m}$. Depth profiles were obtained by sputtering ion beams of $\mathrm{Cs}^{+}(3 \mathrm{keV})$ on a $100 \mu \mathrm{m} \times$ $100 \mu \mathrm{m}$ square. Sputtering rate was measured on a Si wafer as $0.96 \mathrm{~nm} \mathrm{~s}^{-1}$ with a sputtered area of $100 \mu \mathrm{m} \times 100 \mu \mathrm{m}$. AFM (Dimension V equipped with a Nanoscope controller $\mathrm{V}$ and Nanoscope software 7.30, Veeco) was used to measure the elastic modulus of the LLZTO and LLZTO@PAA. The sensitivity and the spring constant of the AFM tip were measured under the contact model and thermal tune model, respectively. A force-strain mapping consisting of $10 \times 10$ points was measured in an area $10 \mu \mathrm{m} \times 10 \mu \mathrm{m}$. The elastic modulus mapping was fitted and plotted using the SPIP (Scanning Probe Image Processor) software. DEMS was used to detect the $\mathrm{H}_{2}$ release and confirm the reaction mechanism. The structure and composition of LiPAA studied by SEM, XPS, FTIR, and Raman.

To investigate the interface between the LLZTO ceramic pellets and Li metal, SEM sample preparation was performed as follows: the Li metal on the LLZTO pellets was melted at $250{ }^{\circ} \mathrm{C}$ for $30 \mathrm{~min}$ and then cooled to room temperature. The LLZTO pellets with $\mathrm{Li}$ metal were fractured using thin-tipped tweezers. Crosssectional samples were chosen for SEM investigation.

To investigate the Li dendrite growing along the grain boundary of LLZTO ceramic pellets, SEM sample preparation was performed as follows: short-circuited cells were disassembled in an Ar-filled glovebox. After completely removing the $\mathrm{Li}$ metal on the LLZTO pellets by sanding, dark spots were observed on the white LLZTO surface, indicating the endpoints of Li dendrite penetration. LLZTO pellets were fractured at dark spots using thin-tipped tweezers. Cross-sectional samples with a black line along grain boundaries were chosen for SEM investigation.

DFT calculations. Calculations were performed using the Vienna ab initio simulation package (VASP) code based on $\mathrm{DFT}^{49,50}$, employing the projector augmented wave (PAW) method as the potentials ${ }^{51}$, and the Perdew-Burke
-Ernzerhof (PBE) generalized-gradient approximation (GGA) as the exchangecorrelation functional ${ }^{52}$. In the model of $\mathrm{PAA} / \mathrm{Li}$, a single chain was designed to attach to $\mathrm{Li}(001)$, the $\mathrm{Li}$ atoms of the innermost layer are fixed to bulk $\mathrm{Li}$, and a $35 \AA$ vacuum slab was built on the Li surface. The DFT-D2 method was employed to incorporate van der Waals interactions between atoms ${ }^{53}$. The $\mathrm{Li}_{2} \mathrm{CO}_{3}(001) / \mathrm{Li}$ (001) and LLZO(110)/Li(001) models were built following refs. ${ }^{54-56}$. For structural relaxation and energy/DOS calculations, an energy cutoff of $520 \mathrm{eV}$ and a $1 \times 1 \times 1$ $\mathrm{K}$-point Monkhorst-Pack grid were used. The convergence criterion for energy and force for structural relaxation were set as $1.0 \times 10^{-5} \mathrm{eV}$ and $0.01 \mathrm{eV} \AA^{-1}$, respectively. All the structures were visualized by VESTA ${ }^{57}$.

Electrochemical performance tests. Ionic conductivity of the LLZTO samples was measured by an impedance analyzer (Novocontrol Beta High Performance Impedance Analyzer) with an AC of $10 \mathrm{mV}$ from 0.1 to $20 \mathrm{MHz}$ in frequency. Thin gold layers were plated on both sides of the ceramic pellets by sputtering to be used as electrodes for conductivity testing. The LLZTO@PAA pellets were sandwiched between two pieces of $\mathrm{Li}$ metal to construct symmetric cells. Li metal electrodes were melted onto the two sides of the LLZTO@PAA pellets at $250{ }^{\circ} \mathrm{C}$ for $30 \mathrm{~min}$ in an Ar-filled glovebox before sealing in Swagelok-type cell molds. A pressure of $\sim 10 \mathrm{~N} \mathrm{~cm}^{-2}$ was exerted on the ceramic plates using springs to maintain good contact. EIS measurements were performed in a frequency range from $1 \mathrm{MHz}$ to $0.1 \mathrm{~Hz}$ with an amplitude of $10 \mathrm{mV}$ by an Autolab instrument. Galvanostatic cycling tests were conducted using a NEWARE battery cycler (CT-4000) using different current densities at $25^{\circ} \mathrm{C}$. Li symmetric cells with other surface modifications were cycled under the same conditions.

The composite cathode was prepared as follows: $0.3 \mathrm{M}$ Lithium bis (trifluoromethanesulfonyl)imide (LiTFSI, Sigma-Aldrich) was dissolved in ionic liquid (IL) (PY14TFSI, Sigma-Aldrich) to obtain a homogeneous IL-0.3 M solution $\mathrm{LiFePO}_{4}$ (LFP), super P conductive additive (SP), polyvinylidene fluoride (PVDF), and IL-0.3 $\mathrm{M}$ with a weight ratio of LCO:SP:PVDF:IL-0.3 $\mathrm{M}=8: 1: 1: 6$ were then ground thoroughly in a mortar. Finally, the slurry was coated on $\mathrm{Al}$ foil to form a composite cathode with an active material loading of $\sim 2 \mathrm{mg} \mathrm{cm}^{-2}$.

\section{Data availability}

The data that support the findings of this study are available from the authors on reasonable request, see author contributions for specific data sets.

Received: 12 July 2020; Accepted: 24 November 2020; Published online: 08 January 2021

\section{References}

1. Tarascon, J.-M. \& Armand, M. Issues and challenges facing rechargeable lithium batteries. Nature 414, 359-367 (2001).

2. Jia, M., Zhao, N., Huo, H. \& Guo, X. Comprehensive Investigation into garne electrolytes toward application-oriented solid lithium batteries. Electrochem. Energy Rev. https://doi.org/10.1007/s41918-020-00076-1 (2020).

3. Dudney, N. J., Bates, J. B., Zuhr, R. A., Luck, C. F. \& Robertson, J. D. Sputtering of lithium compounds for preparation of electrolyte thin-films. Solid State Ion. 53, 655-661 (1992).

4. Kennedy, J. H., Sahami, S., Shea, S. W. \& Zhang, Z. M. Preparation of $\mathrm{Li}_{2} \mathrm{~S}-\mathrm{P}_{2} \mathrm{~S}_{5}$ amorphous solid electrolytes by mechanical milling. Solid State Ion 18-19, 368-371 (1986).

5. Goodenough, J. B., Hong, H. Y. P. \& Kafalas, J. A. Fast $\mathrm{Na}^{+}$-Ion transport in skeleton structures. Mater. Res. Bull. 11, 203-220 (1976).

6. Liu, Y. et al. Stabilizing the interface of NASICON solid electrolyte against Li metal with atomic layer deposition. ACS Appl. Mater. Interfaces 10, 31240-31248 (2018)

7. Inaguma, Y. et al. High ionic-conductivity in lithium lanthanum titanate. Solid State Commun. 86, 689-693 (1993)

8. Li, X. et al. Water-mediated synthesis of a superionic halide solid electrolyte. Angew. Chem. 131, 16579-16584 (2019).

9. Murugan, R., Thangadurai, V. \& Weppner, W. Fast lithium ion conduction in garnet-type $\mathrm{Li}_{7} \mathrm{La}_{3} \mathrm{Zr}_{2} \mathrm{O}_{12}$. Angew. Chem. Int. Ed. 46, 7778-7781 (2007).

10. Thangadurai, V., Pinzaru, D., Narayanan, S. \& Baral, A. K. Fast solid-state Li ion conducting garnet-type structure metal oxides for energy storage. J. Phys. Chem. Lett. 6, 292-299 (2015).

11. Samson, A. J., Hofstetter, K., Bag, S. \& Thangadurai, V. A bird's-eye view of $\mathrm{Li}$-stuffed garnet-type $\mathrm{Li}_{7} \mathrm{La}_{3} \mathrm{Zr}_{2} \mathrm{O}_{12}$ ceramic electrolytes for advanced allsolid-state Li batteries. Energy Environ. Sci. 12, 2957-2975 (2019).

12. $\mathrm{Yu}, \mathrm{S}$. et al. Elastic properties of the solid electrolyte $\mathrm{Li}_{7} \mathrm{La}_{3} \mathrm{Zr}_{2} \mathrm{O}_{12}$ (LLZO). Chem. Mater. 28, 197-206 (2015).

13. Brissot, C., Rosso, M., Chazalviel, J.-N. \& Lascaud, S. Dendritic growth mechanisms in lithium/polymer cells. J. Power Sources 81, 925-929 (1999).

14. Tsai, C.-L. et al. $\mathrm{Li}_{7} \mathrm{La}_{3} \mathrm{Zr}_{2} \mathrm{O}_{12}$ interface modification for $\mathrm{Li}$ dendrite prevention. ACS Appl. Mater. Interfaces 8, 10617-10626 (2016). 
15. Brissot, C., Rosso, M., Chazalviel, J.-N., Baudry, P. \& Lascaud, S. In situ study of dendritic growth inlithium/PEO-salt/lithium cells. Electrochim. Acta 43, 1569-1574 (1998).

16. Cheng, E. J., Sharafi, A. \& Sakamoto, J. Intergranular Li metal propagation through polycrystalline $\mathrm{Li}_{6.25} \mathrm{Al}_{0.25} \mathrm{La}_{3} \mathrm{Zr}_{2} \mathrm{O}_{12}$ ceramic electrolyte. Electrochim. Acta 223, 85-91 (2017).

17. Shen, F., Dixit, M. B., Xiao, X. \& Hatzell, K. B. Effect of pore connectivity on Li dendrite propagation within LLZO electrolytes observed with synchrotron Xray tomography. ACS Energy Lett. 3, 1056-1061 (2018).

18. Swamy, T. et al. Lithium metal penetration induced by electrodeposition through solid electrolytes: example in single-crystal $\mathrm{Li}_{6} \mathrm{La}_{3} \mathrm{ZrTaO}_{12}$ garnet. $J$. Electrochem. Soc. 165, A3648-A3655 (2018).

19. Xiao, Y. et al. Understanding interface stability in solid-state batteries. Nat. Rev. Mater. 5, 105-126 (2019)

20. Han, X. et al. Negating interfacial impedance in garnet-based solid-state Li metal batteries. Nat. Mater. 16, 572-579 (2017).

21. Luo, W. et al. Transition from superlithiophobicity to superlithiophilicity of garnet solid-state electrolyte. J. Am. Chem. Soc. 138, 12258-12262 (2016)

22. Wang, C. et al. Universal soldering of lithium and sodium alloys on various substrates for batteries. Adv. Energy Mater. 8, 1701963 (2017).

23. Huo, $\mathrm{H}$. et al. In-situ formed $\mathrm{Li}_{2} \mathrm{CO}_{3}$-free garnet/Li interface by rapid acid treatment for dendrite-free solid-state batteries. Nano Energy 61, 119-125 (2019).

24. Sharafi, A. et al. Surface chemistry mechanism of ultra-low interfacial resistance in the solid-state electrolyte $\mathrm{Li}_{7} \mathrm{La}_{3} \mathrm{Zr}_{2} \mathrm{O}_{12}$. Chem. Mater. 29, 7961-7968 (2017).

25. Sharafi, A., Meyer, H. M., Nanda, J., Wolfenstine, J. \& Sakamoto, J. Characterizing the $\mathrm{Li}-\mathrm{Li}_{7} \mathrm{La}_{3} \mathrm{Zr}_{2} \mathrm{O}_{12}$ interface stability and kinetics as a function of temperature and current density. J. Power Sources 302, 135-139 (2016).

26. Wang, M. J., Choudhury, R. \& Sakamoto, J. Characterizing the Li-solidelectrolyte interface dynamics as a function of stack pressure and current density. Joule 3, 2165-2178 (2019).

27. Hitz, G. T. et al. High-rate lithium cycling in a scalable trilayer Li-garnet electrolyte architecture. Mater. Today 22, 50-57 (2019).

28. Huo, H. et al. Design of a mixed conductive garnet/Li interface for dendritefree solid lithium metal batteries. Energy Environ. Sci. 13, 127-134 (2020).

29. Han, F. D. et al. High electronic conductivity as the origin of lithium dendrite formation within solid electrolytes. Nat. Energy 4, 187-196 (2019).

30. Xie, X. et al. Lithium expulsion from the solid-state electrolyte $\mathrm{Li}_{6.4} \mathrm{La}_{3} \mathrm{Zr}_{1.4} \mathrm{Ta}_{0.6} \mathrm{O}_{12}$ by controlled electron injection in a SEM. ACS Appl. Mater. Interfaces 10, 5978-5983 (2018).

31. Tian, H.-K., Xu, B. \& Qi, Y. Computational study of lithium nucleation tendency in $\mathrm{Li}_{7} \mathrm{La}_{3} \mathrm{Zr}_{2} \mathrm{O}_{12}$ (LLZO) and rational design of interlayer materials to prevent lithium dendrites. J. Power Sources 392, 79-86 (2018).

32. Tian, H.-K., Liu, Z., Ji, Y., Chen, L.-Q. \& Qi, Y. Interfacial electronic properties dictate Li dendrite growth in solid electrolytes. Chem. Mater. 31, 7351-7359 (2019).

33. Huang, W. et al. Can we find solution to eliminate Li penetration through solid garnet electrolytes? Mater. Today Nano 10, 100075 (2020).

34. Zhao, N. et al. Solid garnet batteries. Joule 3, 1190-1199 (2019).

35. Shao, Y. et al. Drawing a soft interface: an effective interfacial modification strategy for garnet-type solid-state Li batteries. ACS Energy Lett. 3, 1212-1218 (2018).

36. Du, F. et al. All solid state lithium batteries based on lamellar garnet-type ceramic electrolytes. J. Power Sources 300, 24-28 (2015).

37. Chen, Y. et al. Nanocomposite intermediate layers formed by conversion reaction of $\mathrm{SnO}_{2}$ for Li/garnet/Li cycle stability. J. Power Sources 420, 15-21 (2019).

38. Zaharia, A. et al. Bacterial cellulose-poly (acrylic acid-co-N, N'-methylene-bisacrylamide) interpenetrated networks for the controlled release of fertilizers. RSC Adv. 8, 17635-17644 (2018).

39. $\mathrm{Li}, \mathrm{N}$. W. et al. A flexible solid electrolyte interphase layer for long-life lithium metal anodes. Angew. Chem. Int. Ed. Engl. 57, 1505-1509 (2018).

40. Huo, H. et al. $\mathrm{Li}_{2} \mathrm{CO}_{3}$ : a critical issue for developing solid garnet batteries. ACS Energy Lett. 5, 252-262 (2019).

41. Wang, X. et al. Stress-driven lithium dendrite growth mechanism and dendrite mitigation by electroplating on soft substrates. Nat. Energy 3, 227-235 (2018).

42. Young, T. III An essay on the cohesion of fluids. Philos. Trans. R. Soc. Lond. 95, 65-87 (1805).

43. Liu, Y. \& Ning, X.-S. Influence of $\alpha-\mathrm{Al}_{2} \mathrm{O}_{3}$ (0001) surface reconstruction on wettability of $\mathrm{Al} / \mathrm{Al}_{2} \mathrm{O}_{3}$ interface: a first-principle study. Comput. Mater. Sci. 85, 193-199 (2014).

44. He, M., Cui, Z., Chen, C., Li, Y. \& Guo, X. Formation of self-limited, stable and conductive interfaces between garnet electrolytes and lithium anodes for reversible lithium cycling in solid-state batteries. J. Mater. Chem. A 6, 11463-11470 (2018)
45. Nakamura, T., Amezawa, K., Kulisch, J., Zeier, W. G. \& Janek, J. Guidelines for all-solid-state battery design and electrode buffer layers based on chemical potential profile calculation. ACS Appl. Mater. Interfaces 11, 19968-19976 (2019).

46. Raj, R. \& Wolfenstine, J. Current limit diagrams for dendrite formation in solid-state electrolytes for Li-ion batteries. J. Power Sources 343, 119-126 (2017).

47. Huo, H. et al. Rational design of hierarchical "ceramic-in-polymer" and "polymer-in-ceramic" electrolytes for dendrite-free solid-state batteries. Adv. Energy Mater. 9, 1804004 (2019).

48. Huo, H. Y. et al. Anion-immobilized polymer electrolyte achieved by cationic metal-organic framework filler for dendrite-free solid-state batteries. Energy Storage Mater. 18, 59-67 (2019).

49. Kresse, G. \& Furthmüller, J. Efficient iterative schemes for total-energy calculations using a plane-wave basis set. Phys. Rev. B 54, 11169-11186 (1996).

50. Kresse, G. \& Furthmuller, J. Efficiency of ab-initio total energy calculations for metals and semiconductors using a plane-wave basis set. Comput. Mater. Sci. 6, 15-50 (1996).

51. Blochl, P. E. Projector augmented-wave method. Phys. Rev. B 50, 17953-17979 (1994).

52. Burke, K., Perdew, J. P. \& Langreth, D. C. Is the local density approximation exact for short wavelength fluctuations? Phys. Rev. Lett. 73, 1283-1286 (1994).

53. Grimme, S. Semiempirical GGA-type density functional constructed with a long-range dispersion correction. J. Comput. Chem. 27, 1787-1799 (2006).

54. Liu, Z. et al. Interfacial study on solid electrolyte interphase at Li metal anode: implication for Li dendrite growth. J. Electrochem. Soc. 163, A592-A598 (2016).

55. Gao, J. et al. Rational design of mixed electronic-ionic conducting Ti-doping $\mathrm{Li}_{7} \mathrm{La}_{3} \mathrm{Zr}_{2} \mathrm{O}_{12}$ for lithium dendrites suppression. Adv. Funct. Mater. 2001918 (2019).

56. Gao, J. et al. The ab initio calculations on the areal specific resistance of $\mathrm{Li}$ Metal/ $\mathrm{Li}_{7} \mathrm{La}_{3} \mathrm{Zr}_{2} \mathrm{O}_{12}$ interphase. Adv. Theory Simul. 2, 1900028 (2019).

57. Momma, K. \& Izumi, F. VESTA 3 for three-dimensional visualization of crystal, volumetric and morphology data. J. Appl. Crystallogr. 44, 1272-1276 (2011).

58. Feng, W. L. et al. Building an interfacial framework: Li/garnet interface stabilization through a $\mathrm{Cu}_{6} \mathrm{Sn}_{5}$ layer. ACS Energy Lett. 4, 1725-1731 (2019).

59. $\mathrm{Xu}, \mathrm{H}$. et al. Li3N-modified garnet electrolyte for all-solid-state lithium metal batteries operated at 40 degrees C. Nano Lett. 18, 7414-7418 (2018).

\section{Acknowledgements}

The authors would like to thank the National Key R\&D Program of China (Grant No. 2018YFB0104300), the National Natural Science Foundation of China (Grant No. 51771222, 51532002), the Project of Qingdao Leading talents in Entrepreneurship and innovation, the "Taishan Scholars Program", Natural Sciences and Engineering Research Council of Canada (NSERC), Canada Research Chair Program (CRC), and University of Western Ontario.

\section{Author contributions}

H.H. conceived and designed the experimental work and prepared the manuscript; J.G. carried out the DFT simulations; N.Z. fabricated the garnet ceramic pellets; D.Z. helped with the AFM tests; N.G.H. edited the manuscript. X.L. and Y.S. helped with SEM characterizations; J.F. helped with the explanation of lithium dendrite growth. R.L. purchased all the chemicals. X.G. and X.S. supervised the overall project. All authors have given approval to the final version of the manuscript.

\section{Competing interests}

The authors declare no competing interests.

\section{Additional information}

Supplementary information is available for this paper at https://doi.org/10.1038/s41467 020-20463-y.

Correspondence and requests for materials should be addressed to X.G. or X.S.

Peer review information Nature Communications thanks the anonymous reviewers for their contribution to the peer review of this work.

Reprints and permission information is available at http://www.nature.com/reprints

Publisher's note Springer Nature remains neutral with regard to jurisdictional claims in published maps and institutional affiliations. 
(c) (i) Open Access This article is licensed under a Creative Commons Attribution 4.0 International License, which permits use, sharing, adaptation, distribution and reproduction in any medium or format, as long as you give appropriate credit to the original author(s) and the source, provide a link to the Creative Commons license, and indicate if changes were made. The images or other third party material in this article are included in the article's Creative Commons license, unless indicated otherwise in a credit line to the material. If material is not included in the article's Creative Commons license and your intended use is not permitted by statutory regulation or exceeds the permitted use, you will need to obtain permission directly from the copyright holder. To view a copy of this license, visit http://creativecommons.org/ licenses/by/4.0/.

(C) The Author(s) 2021 